\title{
Influence of indium-tin-oxide thin-film quality on reverse leakage current of indium-tin-oxide/n-GaN Schottky contacts
}

\author{
R. X. Wang, ${ }^{\text {a) }}$ S. J. Xu, ${ }^{\text {b) }}$ A. B. Djurišić, C. D. Beling, C. K. Cheung, \\ C. H. Cheung, and S. Fung \\ Department of Physics, The University of Hong Kong, Pokfulam Road, Hong Kong, China \\ D. G. Zhao and H. Yang \\ State Key Laboratory on Integrated Optoelectronics, Institute of Semiconductors, \\ Chinese Academy of Sciences, Beijing 100083, China \\ X. M. Tao \\ Institute of Textiles and Clothing, The Hong Kong Polytechnic University, Kowloon, Hong Kong, China
}

(Received 21 January 2006; accepted 20 June 2006; published online 19 July 2006)

\begin{abstract}
Indium-tin-oxide (ITO) $/ n$-GaN Schottky contacts were prepared by e-beam evaporation at $200{ }^{\circ} \mathrm{C}$ under various partial pressures of oxygen. X-ray photoemission spectroscopy and positron beam measurements were employed to obtain chemical and structural information of the deposited ITO films. The results indicated that the observed variation in the reverse leakage current of the Schottky contact and the optical transmittance of the ITO films were strongly dependent on the quality of the ITO film. The high concentration of point defects at the ITO-GaN interface is suggested to be responsible for the large observed leakage current of the ITO/n-GaN Schottky contacts. (C) 2006 American Institute of Physics. [DOI: 10.1063/1.2227627]
\end{abstract}

As a result of the rapid development of optoelectronic devices, indium tin oxide (ITO) has been used in a great variety of practical devices For example, under suitable fabrication and postdeposition conditions, ITO can be used successfully as either a Schottky or Ohmic contact to $p$ - and $n$-type III-nitride based devices such as ultraviolet photodetectors ${ }^{1}$ and LEDs. ${ }^{2,3}$ Even with these successes, some open issues still remain regarding the influence of ITO preparation conditions on the results. The technique selected for ITO deposition must be optimized since growth conditions strongly affect the microstructures and chemical compositions of the deposited ITO films, ${ }^{4}$ which in turn modify the electrical and optical properties of the ITO films ${ }^{5-7}$ and their specific performance on $\mathrm{GaN}$ devices. ${ }^{8}$

It is well known that the gaseous environment during any deposition can have a large effect on the quality and optoelectrical properties of the ITO film. ${ }^{4,9}$ The oxygen environment is of particular importance with regard to ITO contacts on III-nitrides through the diffusion and reaction of oxygen. For example, the diffusion of oxygen into $\mathrm{GaN}$ from a sapphire substrate ${ }^{10}$ and a $\mathrm{SiO}_{2}$ top layer ${ }^{11}$ is known to have a significant effect on the electrical and optical properties of the GaN film. While this effect has been discussed ${ }^{10,11}$ further investigation is required. In this work we focus on the effect that varying the oxygen partial pressure has on the electrical characteristics of ITO $/ n-G a N$ Schottky contacts. The results show that the higher oxygen partial pressures can improve the quality of the ITO film crystalline structure and that this has the benefit of reducing the reverse leakage current of the ITO/n-GaN Schottky contact and improving the optical transmittance of the ITO.

Lightly Si-doped $n$-type GaN epilayer wafer grown on (0001) sapphire substrate by metal-organic chemical vapor

\footnotetext{
a) Present address: Institute of Textiles and Clothing, The Hong Kong Polytechnic University, Kowloon, Hong Kong, People's Republic of China.

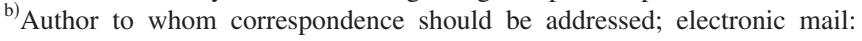
sjxu@hkucc.hku.hk
}

deposition were used for this study. Using Hall measurements, the carrier concentration and mobility of the GaN was found to be $3.3 \times 10^{16} \mathrm{~cm}^{-3}$ and $819 \mathrm{~cm}^{2} \mathrm{~V}^{-1} \mathrm{~s}^{-1}$, respectively. Prior to making the ITO Schottky contact and the In Ohmic contact, the samples were sequentially cleaned by an ultrasonic cleaner with acetone and ethanol, and de-ionized water. ITO films were prepared by e-beam evaporation with the substrate temperature maintained at $200^{\circ} \mathrm{C}$ under different oxygen flow rates from 0 to 6 SCCM (SCCM denotes cubic centimeter per minute at STP). The corresponding partial pressures of oxygen can be found in Table I. Amorphous glass was used as a reference substrate to study the optical transmittance and electrical properties of the ITO films. The transmittance of the ITO films was measured in an HP 8453 UV-visible spectrophotometer system. Current-voltage $(I-V)$ measurements were made using an HP 4155A semiconductor parameter analyzer. The chemical compound information for oxygen, indium, and tin was studied using a PHI 5600 x-ray photoelectron spectroscopy (XPS) system. Variable energy Doppler broadening spectroscopy (VEDBS) measurements were made using the low energy positron beam facility at the University of Hong Kong. ${ }^{12}$

Figure 1 shows typical room-temperature $I-V$ characteristics of the ITO/n-GaN Schottky contacts fabricated under different oxygen partial pressures. It can be seen that all $I-V$ curves clearly exhibit rectification characteristics. However, it is noted that the reverse-biased leakage current of the contacts deposited with higher than 3 SCCM oxygen flow rates is much smaller as compared with contacts deposited with lower or zero oxygen flow rate. Based on thermionicemission theory the Schottky parameters are extracted by fitting the forward exponential section of the formula ${ }^{13}$

$$
I=A A^{*} T^{2} e^{-q \phi_{b} / k T}\left[e^{q V / n k T}-1\right],
$$

where $A, A^{*}, \phi_{b}$, and $n$ are the contact area, the Richardson constant, the Schottky barrier height, and the ideality factor, respectively. The values of $\phi_{b}$ and $n$ are determined (as given in Table I). In sharp contrast to the substantial variation of 
TABLE I. Properties of devices and ITO films deposited at $200{ }^{\circ} \mathrm{C}$.

\begin{tabular}{|c|c|c|c|c|c|}
\hline Sample labels & a & b & $\mathrm{c}$ & $\mathrm{d}$ & $\mathrm{e}$ \\
\hline Oxygen flow rate (SCCM) & 0 & 1 & 3 & 5 & 6 \\
\hline Oxygen partial pressure $\left(\times 10^{-3}\right.$ torr $)$ & 0 & 1.3 & 3.6 & 6.2 & 7.9 \\
\hline Barrier height $(\mathrm{eV})$ & $0.63 \pm 0.003$ & $0.67 \pm 0.03$ & $0.77 \pm 0.04$ & $0.75 \pm 0.03$ & $0.77 \pm 0.02$ \\
\hline Ideality factor $n$ & $2.10 \pm 0.91$ & $1.75 \pm 0.38$ & $1.87 \pm 0.65$ & $1.70 \pm 0.42$ & $1.75 \pm 0.69$ \\
\hline Reverse leakage current at $-10 \mathrm{~V}$ & & & & & \\
\hline$(\mu \mathrm{A})$ & 2300 & 91.5 & 0.031 & 0.073 & 0.078 \\
\hline $\mathrm{Sn} / \mathrm{In}$ ratio $(\%)$ & 27 & 15 & 12 & $\ldots$ & 12 \\
\hline $\mathrm{Sn} / \mathrm{O}+\mathrm{In}+\mathrm{Sn}$ ratio $(\%)$ & 6 & 4 & 4 & $\ldots$ & 3 \\
\hline Concentration $\left(\times 10^{21} \mathrm{~cm}^{-3}\right)$ & 4.01 & 2.58 & 1 & 4.85 & 5.72 \\
\hline Mobility $\left(\mathrm{cm}^{2} / \mathrm{V} \mathrm{s}\right)$ & 2.62 & 11.2 & 4.13 & 4.72 & 4.37 \\
\hline Sheet resistance $(\Omega / \square)$ & 29.9 & 10.8 & 75.5 & 136.5 & 124 \\
\hline
\end{tabular}

the reverse leakage current, the values of $\phi_{b}$ and $n$ are found to be not very sensitive to the oxygen partial pressure. Our results certainly seem consistent with Hsu et al.'s. ${ }^{14}$ As will be shown later, the reverse bias leakage current in our ITO/ $n$-GaN Schottky contacts is strongly correlated with the defects in the ITO films, in particular, defects at the ITO-GaN interface.

Figure 2 shows the transmittance curves of the ITO films grown under different oxygen partial pressures. It is observed that the transmittance of the ITO films deposited under high oxygen partial pressure is much higher than that of the films deposited under zero or low oxygen partial pressure. Moreover, the transmittance edge of the films fabricated under high oxygen partial pressure shifts to shorter wavelengths as compared with that of films deposited without any oxygen flow.

To explore and investigate the reasons for the oxygen environment influencing the reverse-biased characteristic and optical transmittance, an XPS study was performed. Figure 3 shows the XPS Sn $3 d$, In $3 d$, and O $1 s$ data for the films grown under different oxygen partial pressures. It can be seen that the relative intensity of Sn decreases for higher oxygen partial pressures. The integrated intensity ratios $\mathrm{Sn} / \mathrm{In}$ and $\mathrm{Sn} / \mathrm{In}+\mathrm{Sn}+\mathrm{O}$, as obtained from the XPS data, are given in Table I. The ratios of films deposited under low or zero oxygen partial pressure are almost twice that of the ones deposited under high oxygen partial pressure. This change in Sn ratio follows the change seen in the optical transmittance result as mentioned above in which the absorption edge shifts to the lower energy side under excessive Sn. This result agrees with that reported by Kim et al. ${ }^{5}$

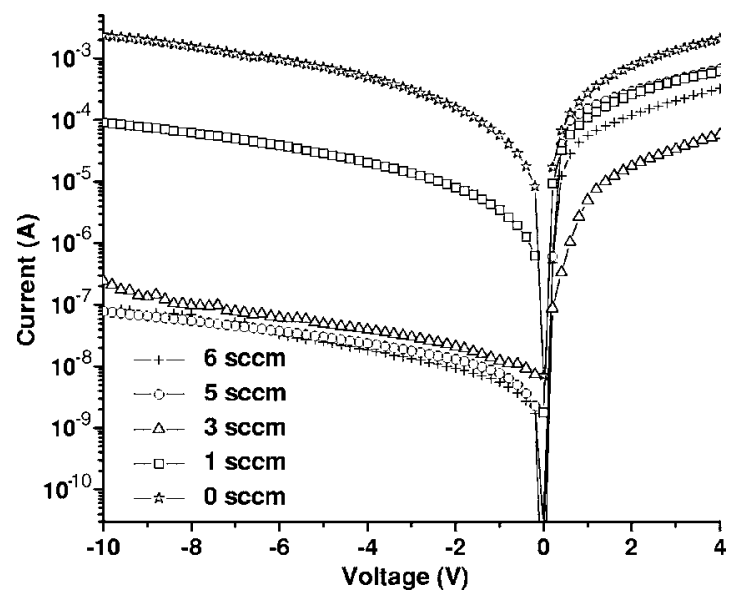

FIG. 1. Typical $I-V$ characteristics of ITO $/ n-G a N$ Schottky contacts fabricated under different partial pressures of oxygen.
Sn acts as a cationic dopant when it substitutes for In and is surrounded by $\operatorname{In}_{2} \mathrm{O}_{3}$. As such, it provides an electron to the conduction band making the ITO conducting. However, too high a $\mathrm{Sn} / \mathrm{In}$ ratio negatively influences the electrical properties of ITO film. ${ }^{4,6}$ A number of suggestions have been made to account for this behavior., ${ }^{4,6,15}$ (1) When one of the nearest-neighbor $\operatorname{In}_{2} \mathrm{O}_{3}$ sites around the $\mathrm{Sn}$ ion is occupied with a second $\mathrm{Sn}$ ion, the donor action is compensated. (2) If excess Sn doping occupies an interstitial site then it forms a deep level electron trap rather than an electron donor and (3) some other hitherto unknown reason exists for $\mathrm{Sn}$ being electrically inactive in amorphous microstructure. In our case, the $\mathrm{Sn} / \mathrm{In}$ weight ratio in the source was $10 \%$ and the substrate temperature during ITO film deposition was $200{ }^{\circ} \mathrm{C}$ which was around the ITO crystallization temperature. ${ }^{16}$ It is thus that the microstructure of the ITO film would be polycrystalline ${ }^{16}$ which places the Sn doping in a regime where behavior is difficult to interpret. Interestingly, it is noted that for zero and low oxygen partial pressures, the sheet resistance of ITO film decreases with increasing oxygen partial pressure. This can best be understood as the primary Sn dopant effect. ${ }^{17}$ However, for higher oxygen partial pressures, the sheet resistance of ITO film increases with increasing oxygen flow rate. One possible explanation for this effect is the decrease in the concentration of oxygen vacancies. ${ }^{7}$

To test the idea that there exist defects that form deep level traps and to investigate the crystal structure of the ITO film and the interface between ITO and GaN, positron beam measurements were performed. Figure 4 shows the VEDBS scans taken on the different contacts grown under different partial pressures of oxygen. The low electron momentum $S$ parameter is usually higher in material that contains a high

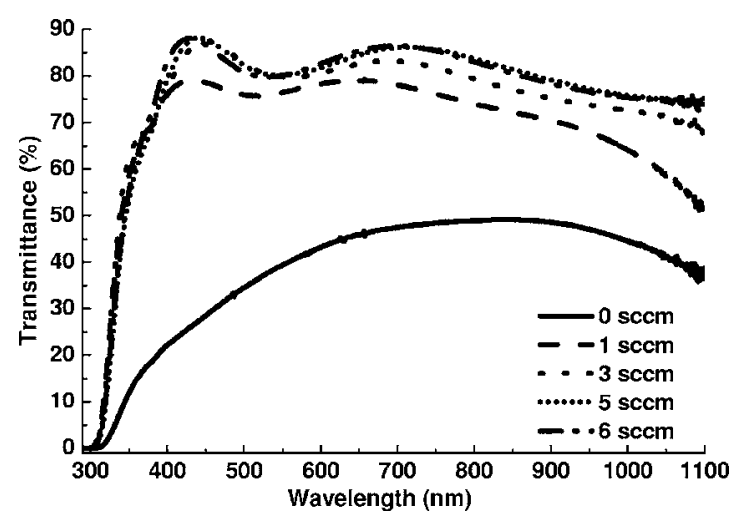

FIG. 2. Transmittance of ITO film deposited under different partial pressures of oxygen. 


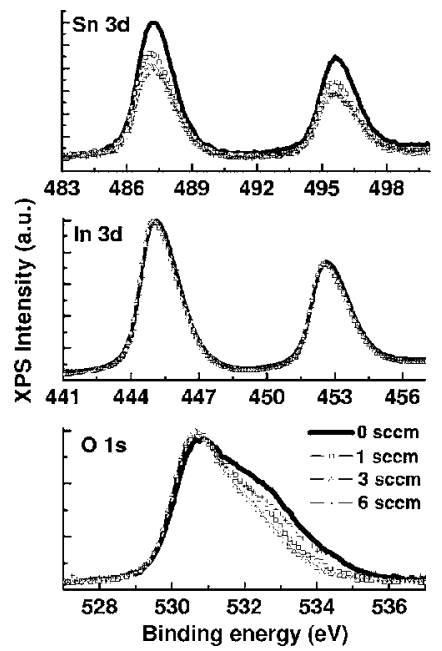

FIG. 3. Sn $3 d$ (top), In $3 d$ (middle), and O $1 s$ (bottom) normalized XPS curves of the ITO films deposited on GaN wafer under different partial pressures of oxygen.

concentration of open volume point defects. This is a result of the positron trapping into open volume defect sites where it predominantly samples low momentum valence electrons. The VEDBS data in the $0-10 \mathrm{keV}$ implantation energy range are indicative of annihilations occurring in the ITO overlayer. At $10 \mathrm{keV}$, the positron beam is maximally sampling the ITO-GaN interface while at higher energies, the $S$ parameter drops to the value characterizing the GaN film. The remarkable feature of this positron annihilation data is that the ITO $S$ parameter is significantly higher in the zero oxygen partial pressure case and drops with increasing oxygen partial pressure. This clearly supports the oxygen vacancy model, oxygen vacancies being more predominant in the films grown without oxygen. Also noted, particularly in the zero oxygen case a peaking in the $S$ parameter is a peaking at the ITO/GaN interface. This indicates the presence of vacancy-type defects at the interface. This peaking phenomenon seems to reduce under higher oxygen partial pressures, presumably as a result of the point defects being removed under oxygenation.

The above evidence for the presence of oxygen vacancy defects both in the ITO film and at the ITO-GaN interface can lend some understanding to the observed pattern of reverse leakage current. The reverse leakage current expected for an ideal ITO/GaN Schottky contact can be estimated using the standard equation $A A^{*} T^{2} e^{-q \phi_{b} / k T}$ and the Richardson constant $A^{*}$ which may be taken as $2.64 \times 10^{5} \mathrm{~A} \mathrm{~m}^{-2} \mathrm{~K}^{-2}$ for $\mathrm{GaN}{ }^{18}$ The values obtained in this way are much smaller than the measured reverse leakage current. This indicates that for contacts made under zero or low oxygen concentration, the oxygen vacancy defects can act as carriergeneration centers at the interface causing larger than expected leakage current. The real picture is likely to be more complicated with other types of point defects making contributions to the generation and tunneling currents. Miller et $a l .{ }^{19}$ have shown that, at higher temperatures, the reverse leakage current of $\mathrm{Ni} / n-\mathrm{GaN}$ Schottky contact is mainly due to a two-step, trap-assisted tunneling. For our case, it is also possible that the increased crystallinity and oxygen content of the ITO film under high oxygen partial pressure results in forming a $\mathrm{GaO}_{x}$ insulator layer ${ }^{8}$ that can reduce tunneling.

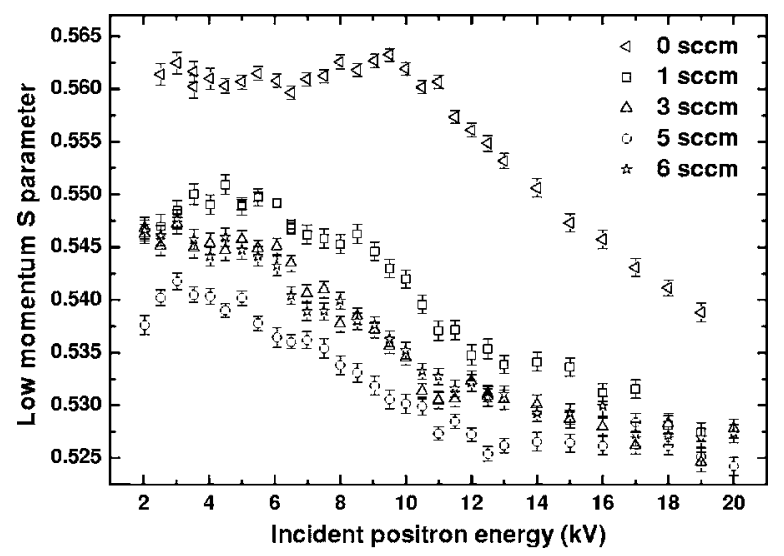

FIG. 4. Positron beam VEDBS scans of the ITO/GaN Schottky contacts deposited under different oxygen partial pressures.

In conclusion, ITO/GaN Schottky contacts fabricated under different oxygen partial pressures have been studied by $I-V$, XPS, and positron beam techniques. Contacts grown under zero or low oxygen partial pressure show poor reverse characteristics. This finding has been related to the higher $\mathrm{Sn} / \mathrm{In}$ ratio found in low oxygenated films and the poor ITO crystallinity. The higher concentration of point defects (most likely oxygen vacancies) as seen through positron annihilation is a source of increased generation current in the Schottky contacts exhibiting high reverse leakage current.

This work was supported by the HKSAR RGC CERG grants under Contract Nos. HKU7036/03P and HKU7021/ 04P. Financial support from the University Development Fund grant (administrated by The University of Hong Kong) is also acknowledged.

${ }^{1}$ C. H. Chen, S. J. Chang, Y. K. Su, G. C. Chi, J. Y. Chi, C. A. Chang, J. K. Sheu, and J. F. Chen, IEEE Photonics Technol. Lett. 13, 848 (2001).

${ }^{2}$ D. W. Kim, H. Y. Lee, M. C. Yoo, and G. Y. Yeom, Appl. Phys. Lett. 86, 052108 (2005).

${ }^{3}$ C. S. Chang, S. J. Chang, Y. K. Su, Y. C. Lin, Y. P. Hsu, S. C. Shei, S. C. Chen, C. H. Liu, and U. H. Liaw, Semicond. Sci. Technol. 18, L21 (2003).

${ }^{4}$ M. Rottmann, H. Hennig, B. Ziemer, R. Kalahna, and K. H. Heckner, J. Mater. Sci. 31, 6465 (1996).

${ }^{5}$ H. Kim, C. M. Gilmore, A. Pique, J. S. Horwitz, H. Mattoussi, H. Murata,

Z. H. Kafafi, and D. B. Chrisey, J. Appl. Phys. 86, 6451 (1999).

${ }^{6}$ F. O. Adurodija, H. Izumi, T. Ishihara, H. Yoshioka, and M. Motoyama, J. Appl. Phys. 88, 4175 (2000).

${ }^{7}$ W. G. Haines and R. H. Bube, J. Appl. Phys. 49, 304 (1978).

${ }^{8}$ J. K. Sheu, Y. K. Su, G. C. Chi, M. J. Jou, and C. M. Chang, Appl. Phys. Lett. 72, 3317 (1998).

${ }^{9}$ W. F. Wu and B. S. Chiou, Semicond. Sci. Technol. 11, 196 (1996).

${ }^{10}$ J. E. Van Nostrand, J. Solomon, A. Saxler, Q. H. Xie, D. C. Reynolds, and D. C. Look, J. Appl. Phys. 87, 8766 (2000).

${ }^{11}$ X. C. Wang, S. J. Xu, S. J. Chua, K. Li, X. H. Zhang, Z. H. Zhang, K. B. Chong, and X. Zhang, Appl. Phys. Lett. 74, 818 (1999).

${ }^{12}$ P. Y. Kuan, S. Fung, and C. D. Beling, Appl. Surf. Sci. 194, 32 (2002).

${ }^{13}$ S. M. Sze, Physics of Semiconductor Devices, 2nd ed. (Wiley, New York, 1981).

${ }^{14}$ J. W. P. Hsu, M. J. Manfra, D. V. Lang, S. Richter, S. N. G. Chu, A. M. Sergent, R. N. Kleiman, L. N. Pfeiffer, and R. J. Molnar, Appl. Phys. Lett. 78, 1685 (2001).

${ }^{15}$ H. Kim, C. M. Gilmore, A. Piqué, J. S. Horwitz, H. Mattoussi, H. Murata, Z. H. Kafafi, and D. B. Chrisey, J. Appl. Phys. 86, 6451 (1999).

${ }^{16}$ R. X. Wang, C. D. Beling, S. Fung, A. B. Djurišić, C. C. Ling, C. Kwong, and S. Li, J. Phys. D 38, 2000 (2005).

${ }^{17}$ S. Noguchi and H. Sakata, J. Phys. D 14, 1523 (1981).

${ }^{18}$ A. C. Schmitz, A. T. Ping, M. Asif Khan, Q. Chen, J. W. Yang, and I. Adesida, Semicond. Sci. Technol. 11, 1464 (1996).

${ }^{19}$ E. J. Miller, E. T. Yu, P. Waltereit, and J. S. Speck, Appl. Phys. Lett. 84, 535 (2004). 
Applied Physics Letters is copyrighted by the American Institute of Physics (AIP). Redistribution of journal material is subject to the AIP online journal license and/or AIP copyright. For more information, see http://ojps.aip.org/aplo/aplcr.jsp 